\title{
Frequency Variation of the AC Order Parameter Susceptibility of the Metamagnetic Ising Model
}

\author{
Gul Gulpinar \\ Department of Physics, Dokuz Eylül University, 35160 Izmir, Turkey \\ Correspondence should be addressed to Gul Gulpinar; gul.gulpinar@deu.edu.tr
}

Received 18 January 2018; Revised 16 February 2018; Accepted 26 February 2018; Published 18 April 2018

Academic Editor: Yuri Galperin

Copyright ( 2018 Gul Gulpinar. This is an open access article distributed under the Creative Commons Attribution License, which permits unrestricted use, distribution, and reproduction in any medium, provided the original work is properly cited.

\begin{abstract}
The extensive investigation of the absorptive and reactive parts of the AC order parameter susceptibility spectra of iron group dihalides, which is obtained on the basis of Onsager theory of irreversible processes, revealed the fact that the diagonal phenomenological rate coefficients $\gamma_{s}$ and $\gamma_{m}$ have an important impact on the nature of the order parameter relaxation process. The number of the relaxation peaks appearing in the double logarithmic plots of $\chi_{s}^{\prime \prime}$ versus field frequency $\omega$ and the number of plateau regions in $\chi_{s}^{\prime}$ spectrum depends on the values of $\gamma_{s}$ and $\gamma_{m}$. Only for $\left|\gamma_{s}\right| \gg\left|\gamma_{m}\right|$ does the relaxation evolve from a simple Debye exponential at high temperatures to a two-step process at lower temperatures in which there exist two long relaxation times characterizing the relaxation of staggered magnetization. In parallel with these characteristics of the order parameter relaxation, Cole-Cole plots $\left(\chi_{s}^{\prime \prime}-\chi_{s}^{\prime}\right)$ are shown to consist of two arcs in the metamagnetic phase and of a semicircle in the paramagnetic phase.
\end{abstract}

\section{Introduction}

If a magnetic system is perturbed by an alternative current (AC) external magnetic field, magnetic properties of the system that are coupled to the magnetic field change accordingly. This fact lets the investigation of the AC susceptibility play an important role in the study of magnetic phase transitions and spin dynamics [1-4]. The AC susceptibility of correlated spin structures consisting of clusters or domains of various volumes is generally described by models based on linear response theory which assumes a single relaxation time or a distribution of relaxation times that measure how fast the system approaches equilibrium after a disturbance [5-7]. In the phenomenological spin-glass model prescribing a wide distribution of relaxation times, it has been reported that the imaginary part of the AC susceptibility is related to the density of relaxation times [6-8].

Metamagnetic systems are systems that have both ferromagnetic and antiferromagnetic couplings, simultaneously. They attracted much interest due to the fact that it is possible to induce novel kinds of critical behavior by forcing competition between these couplings, especially by applying a magnetic field $[9,10]$. Compounds such as $\mathrm{FeCl}_{2}, \mathrm{FeBr}_{2}$, $\mathrm{NiCl}_{2}, \mathrm{FeMgBr}_{2}, \mathrm{CoCl}_{2}$, and $\mathrm{FeCl}_{2} \cdot 2 \mathrm{H}_{2} \mathrm{O}$ are grouped as metamagnetic substances [11]. The transitions in these materials are so-called field induced transitions which are distinguished from other magnetization processes. In particular, $\mathrm{FeCl}_{2}$ and $\mathrm{FeBr}_{2}$ are well-known Ising type metamagnets [9]. The spins of the iron ions display a ferromagnetic order in the triangular layers, perpendicular to the $c$-axis of the crystals, with the sign of the magnetization per layer changing layer by layer: say an "up (+)" layer is followed by a "down (-)" layer in the metamagnetic phase. If one applies an external physical magnetic field $H$, one type of layer (i.e., "up") is favored, and $H$ keeps on increasing, eventually the magnetization will be equal in all layers [12]. The corresponding phase transition is of the first order at low temperatures, while it is continuous at higher temperatures and low fields.

The aim of this study is to formulate the steady-state dynamics and investigate the frequency variance of the AC order parameter susceptibility of a metamagnetic Ising system via the phenomenological kinetic coefficients and the linear response theory. In particular, the frequency behavior of the AC staggered susceptibility of the metamagnetic Ising model near the field induced Neel point will be studied. The critical and multicritical characteristics of the relaxation times of the model are presented by studying the relaxation dynamics $[13,14]$. Recently, only the temperature variations 
of the static and dynamic magnetic response functions for the same model Hamiltonian have been presented by studying the steady-state solutions of the kinetic equations in the existence of oscillating staggered field [15]. The AC susceptibility obtained by means of linear response theory is independent of the amplitude but dependent on the frequency $\omega$ of the AC field. In addition, even though the temperature dependence and critical exponents of the real and imaginary parts of the AC susceptibility characterize the relaxation process of a spin system near the critical point, the temperature variance is not a good criterion to detect the existence of long relaxation times which characterize relaxation of the order parameter [16].

A brief discussion of the mean-field equilibrium behavior of the model is given in Section 2. Section 3 is devoted to the derivation of the relaxation times and the frequency dependent AC order parameter susceptibility, $\chi_{s}(\omega)$. The AC order parameter susceptibility spectra and Cole-Cole plots $\left(\chi_{s}^{\prime \prime}-\chi_{s}^{\prime}\right)$ are presented near the critical point in Section 4. Finally, a summary and discussion of the results are given in the last section.

\section{Equilibrium Properties of the Model}

The metamagnetic Ising model on a simple cubic lattice, setting the lattice constant equal to one, can be described by the Hamiltonian

$$
\begin{aligned}
\widehat{H}= & -J_{i j} \sum_{\text {inter }} S_{i} S_{j}-J_{k l}^{\prime} \sum_{\text {intra }} S_{k} S_{l}-H \sum_{\text {sites }} S_{i} \\
& -H_{s} \sum_{\text {sites }}(-1)^{i} S_{i},
\end{aligned}
$$

where Ising spin at site $i$ can take one of two values $\left(S_{i}=\right.$ $\pm 1)$ depending on whether the spin is "up" or "down," respectively. The first sum refers to couplings $J_{i j}$ between spins $S_{j}$ and $S_{j}$ in the same $x$ - $y$ layers, the second sum denotes interactions of spins in adjacent layers, and the physical and staggered fields $H$ and $H_{s}$ act on all spins. In this spin system, there exist antiferromagnetic interlayer couplings, $J_{k l}^{\prime}=$ $J_{1}<0$, and ferromagnetic intralayer interactions between neighboring spins, $J_{i j}=J_{2}>0$.

The reference spin, at site $(x, y, z)$, interacts only with its two nearest neighbors, at sites $(x, y, z-1)$ and $(x, y, z+1)$ in the adjacent layers above and below, with the coupling $J_{k l}^{\prime}=$ $J_{1}<0$. In addition, it couples to its four nearest neighbors in its layer located at $(x-1, y, z),(x+1, y, z),(x, y-1, z)$, and $(x, y+1, z)$ via ferromagnetic intralayer exchange coupling $J_{i j}=J_{2}>0$. Due to the existence of the antiferromagnetic coupling, it is convenient to divide the system into two sublattices ( $a$ and $b$ ) and define magnetization of each sublattice as $M_{a}=\left\langle S_{i}\right\rangle_{a}$ and $M_{b}=\left\langle S_{j}\right\rangle_{b}$. In addition, one should introduce total and staggered magnetization as follows:

$$
\begin{gathered}
m=\frac{M_{a}+M_{b}}{2}, \\
s=\frac{M_{a}-M_{b}}{2} .
\end{gathered}
$$

The mean-field free energy of the system can be expressed as follows:

$$
\begin{aligned}
\Psi & \left(m, s, T, H, H_{s}\right)=\frac{1}{2} N J_{1} z_{1}\left(m^{2}-s^{2}\right)-\frac{1}{2} N J_{2} z_{2}\left(s^{2}\right. \\
& \left.+m^{2}\right)-\frac{1}{4} N k_{B} T(4 \ln (2) \\
& -(1+s+m) \ln (1+s+m) \\
& -(1-s-m) \ln (1-s-m) \\
& -(1+m-s) \ln (1+m-s) \\
& -(1-m+s) \ln (1-m+s))-N H \mu_{B} g m \\
& -N H_{s} \mu_{B} g s,
\end{aligned}
$$

where $N, T, k_{B}, \mu_{B}, g, N, z_{1}, z_{2}$ are the number of spins, temperature, Boltzmann constant, Bohr magneton, spin factor, total number of spins, and coordination numbers, respectively $[9,13]$. As discussed above, $z_{1}=4$ and $z_{2}=2$. Using the minimization conditions of equilibrium

$$
\begin{aligned}
& \frac{\partial \Psi}{\partial m}=0, \\
& \frac{\partial \Psi}{\partial s}=0,
\end{aligned}
$$

the following self-consistent equations are obtained:

$$
\begin{aligned}
& m \\
& =\frac{\sinh \left(2(H-\kappa m) / k_{B} T\right)}{\cosh \left(2(H-\kappa m) / k_{B} T\right)+\cosh \left(2\left(H_{s}+\theta s\right) / k_{B} T\right)}, \\
& s \\
& =\frac{\sinh \left(2\left(H_{s}+\theta s\right) / k_{B} T\right)}{\cosh \left(2(H-\kappa m) / k_{B} T\right)+\cosh \left(2\left(H_{s}+\theta s\right) / k_{B} T\right)} .
\end{aligned}
$$

Here, $\kappa=J_{1}-J_{2}$ and $\theta=J_{1}+J_{2}$.

As one converges to critical point, the staggered magnetization $(s)$ vanishes whereas the total magnetization $(m)$ stays finite [12]. Thus, $s$ is the order parameter of the metamagnetic Ising model. In the extensive theoretical review by Kincaid and Cohen, it has been reported that, depending on the ratio of the antiferromagnetic and ferromagnetic exchange interactions $\left(r=J_{2} /\left|J_{1}\right|\right)$, metamagnetic Ising model exhibits different kinds of phase diagrams in $(H, T)$ plane for $H_{s}=0$.

(i) If $r>0.6$, then the phase transition between the antiferromagnetic and paramagnetic phases is discontinuous at low temperatures and strong fields while it is continuous at higher temperature regime. First- and second-order transition lines are joined at the tricritical point (see Figure 1(a) of [13]).

(ii) If $0<r<0.6$, then the topology of the phase diagram changes in nature: the transitions between the antiferromagnetic and paramagnetic phases are of the first order at low temperatures and strong magnetic fields while they are of the second order at high temperature regions. In addition, the 
phase diagram displays reentrance: a portion of the secondorder transition line is masked by a first-order transition line which gives rise to an appearance of a critical endpoint where the second-order line meets two first-order lines, one of which is immersed in the ordered phase and ends at a double critical endpoint (see Figure 1(b) of [13]).

\section{Derivation of AC Order Parameter Susceptibility}

In this section, we will first discuss the relaxation behavior of the metamagnetic Ising model by investigating the relaxation process which takes place if there exists a small disturbance that removes the system slightly from equilibrium. Then, we will study the steady-state response of the system to an oscillating staggered magnetic field, which will let us obtain the frequency dependent AC order parameter susceptibility.

\subsection{Free Energy Production, Rate Equations, and Relaxation} Times. It is well known that the metamagnetic-paramagnetic phase transition lines occur at places which are away from the $H=0$ axis. Since then, we have assumed that there exist small deviations in the physical and staggered magnetic fields $\left(\delta H=H-H_{0}, \delta H_{s}=H_{s}-H_{s_{0}}\right)$ only for a short while, which removes the system slightly from equilibrium [9]. The near-equilibrium free energy of the system can be expressed as follows:

$$
\Psi\left(m, s, T, H, H_{s}\right)=\Psi_{0}\left(m_{0}, s_{0}, T, H_{0}, H_{s_{0}}\right)+\Delta \Psi,
$$

where $\Psi_{0}$ is the equilibrium free energy and $\Delta \Psi$ is the production of the free energy due to the variance of the external fields. The free energy production for the metamagnetic Ising model reads

$$
\begin{aligned}
\Delta \Psi= & a\left(H-H_{0}\right)+b\left(H_{s}-H_{s_{0}}\right)+\frac{1}{2} c\left(m-m_{0}\right)^{2} \\
& +\frac{1}{2} d\left(s-s_{0}\right)^{2}+\frac{1}{2} e\left(H-H_{0}\right)^{2} \\
& +\frac{1}{2} f\left(H_{s}-H_{s_{0}}\right)^{2}+g\left(m-m_{0}\right)\left(s-s_{0}\right) \\
& +h\left(m-m_{0}\right)\left(H-H_{0}\right) \\
& +i\left(m-m_{0}\right)\left(H_{s}-H_{s_{0}}\right)+j\left(s-s_{0}\right)\left(H-H_{0}\right) \\
& +k\left(s-s_{0}\right)\left(H_{s}-H_{s_{0}}\right),
\end{aligned}
$$

where

$$
\begin{aligned}
& a=\left(\frac{\partial \Psi}{\partial H}\right)_{\mathrm{eq}}=-N \mu_{B} m_{0}, \\
& b=\left(\frac{\partial \Psi}{\partial H_{s}}\right)_{\mathrm{eq}}=-N \mu_{B} s_{0} \\
& c=\left(\frac{\partial^{2} \Psi}{\partial m^{2}}\right)_{\mathrm{eq}}
\end{aligned}
$$

$$
\begin{aligned}
& =N\left\{J_{1}-J_{2}+\frac{k_{B} T}{4}\left(\frac{1}{1+s_{0}+m_{0}}+\frac{1}{1-s_{0}-m_{0}}\right.\right. \\
& \left.\left.+\frac{1}{1+m_{0}-s_{0}}+\frac{1}{1-m_{0}+s_{0}}\right)\right\} \text {, } \\
& d=\left(\frac{\partial^{2} \Psi}{\partial s^{2}}\right)_{\mathrm{eq}} \\
& =-N\left\{J_{1}+J_{2}+\frac{k_{B} T}{4}\left(\frac{1}{1+s_{0}+m_{0}}+\frac{1}{1-s_{0}-m_{0}}\right.\right. \\
& \left.\left.+\frac{1}{1+m_{0}-s_{0}}+\frac{1}{1-m_{0}+s_{0}}\right)\right\} \text {, } \\
& e=\left(\frac{\partial^{2} \Psi}{\partial H^{2}}\right)_{\mathrm{eq}}=0 \\
& f=\left(\frac{\partial^{2} \Psi}{\partial H_{s}^{2}}\right)_{\mathrm{eq}}=0 \\
& g=\left(\frac{\partial^{2} \Psi}{\partial m \partial s}\right)_{\mathrm{eq}}=\frac{N k_{B} T}{4}\left(\frac{1}{1+s_{0}+m_{0}}+\frac{1}{1-s_{0}-m_{0}}\right. \\
& \left.-\frac{1}{1+m_{0}-s_{0}}-\frac{1}{1-m_{0}+s_{0}}\right) \text {, } \\
& h=\left(\frac{\partial^{2} \Psi}{\partial H \partial m}\right)_{\mathrm{eq}}=-N \mu_{B} \text {, } \\
& i=\left(\frac{\partial^{2} \Psi}{\partial m \partial H_{s}}\right)_{\mathrm{eq}}=0, \\
& j=\left(\frac{\partial^{2} \Psi}{\partial H \partial s}\right)_{\mathrm{eq}}=0, \\
& k=\left(\frac{\partial^{2} \Psi}{\partial H_{s} \partial s}\right)_{\mathrm{eq}}=-N \mu_{B},
\end{aligned}
$$

which are the so-called free energy production coefficients. The time derivatives of the staggered and total magnetization are treated as generalized currents conjugate to their appropriate generalized forces in the realm of the theory of irreversible thermodynamics. One obtains the generalized forces $X_{m}$ and $X_{s}$ conjugate to the generalized currents $\dot{m}$ and $\dot{s}$ by differentiating $\Delta \Psi$ with respect to $m-m_{0}$ and $s-s_{0}$ :

$$
\begin{aligned}
X_{m}= & c\left(m-m_{0}\right)+g\left(s-s_{0}\right)+h\left(H-H_{0}\right) \\
& +i\left(H_{s}-H_{s_{0}}\right), \\
X_{s}= & d\left(s-s_{0}\right)+g\left(m-m_{0}\right)+j\left(H-H_{0}\right) \\
& +k\left(H_{s}-H_{s_{0}}\right) .
\end{aligned}
$$

The relations between the currents and forces may be expressed in terms of a matrix of the phenomenological rate coefficients. Since both $m$ and $s$ are odd variables under time 
inversion, this matrix should be symmetric $[13,17]$. Thus, the off-diagonal elements will be identical:

$$
\left[\begin{array}{c}
\dot{m} \\
\dot{s}
\end{array}\right]=\left[\begin{array}{cc}
\gamma_{m} & \gamma \\
\gamma & \gamma_{s}
\end{array}\right]\left[\begin{array}{c}
X_{m} \\
X_{s}
\end{array}\right] .
$$

One should consider the corresponding homogeneous equations resulting when there is no external stimulation $\left(H=H_{0}\right.$ and $\left.H_{s}=H_{s_{0}}\right)$ in order to obtain two relaxation times that will govern the relaxation dynamics of the system. Then, the matrix equation given by (9) becomes

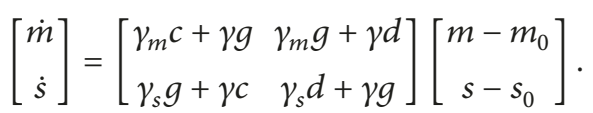

One can observe from (11) that the off-diagonal rate coefficient $(\gamma)$ couples the total and staggered magnetization currents. The kinetic equations given by (10) can be solved by assuming a form for the solution $\left(m-m_{0}, s-s_{0} \simeq e^{-t / \tau}\right)$ by making use of the secular equation given below:

$$
\left|\begin{array}{cc}
\tau^{-1}+\gamma_{m} c+\gamma g & \gamma_{m} g+\gamma d \\
\gamma_{s} g+\gamma c & \tau^{-1}+\gamma_{s} d+\gamma g
\end{array}\right|=0 .
$$

The resulting reciprocal relaxation times can be found as

$$
\begin{aligned}
& \frac{1}{\tau_{1}}=\frac{1}{2}\left(\sqrt{\Delta}-2 \gamma g-\gamma_{s} d-\gamma_{m} c\right), \\
& \frac{1}{\tau_{2}}=-\frac{1}{2}\left(\sqrt{\Delta}+2 \gamma g-\gamma_{s} d+\gamma_{m} c\right),
\end{aligned}
$$

where $\Delta$ is given as follows:

$$
\begin{aligned}
\Delta= & 4 \gamma_{s} \gamma d g+4 \gamma_{m} \gamma g c+\gamma_{s}^{2} d^{2}+\gamma_{m}^{2} c^{2}+4 \gamma^{2} d c \\
& +2 \gamma_{m} \gamma_{s}\left(2 g^{2}-c d\right) .
\end{aligned}
$$

We should emphasize that, for temperatures near the Neel temperature, the following assumption is valid:

$$
\gamma_{m} \gamma_{s}-\gamma^{2} \approx \gamma_{m} \gamma_{s} .
$$

In other words, we do not take into account the interference between the relaxation processes of staggered and total magnetization. Temperature variances of the relaxation times for finite external magnetic field values in the ordered and disordered phases for the Onsager rate coefficient sets satisfying the assumption (see (15)) were discussed in [13]. The findings of this study can be summarized as follows: for continuous phase transitions, $\tau_{1}$ scarcely varies with temperature in the ordered phase and rises slightly just below and above the phase transition temperatures. It should be noted that cusps are observed for the critical behavior of $\tau_{1}$, whereas $\tau_{2}$ increases rapidly with rising temperature and diverges near the second- and the higher-order phase transition points as $\left|T-T_{N}\right|^{-1}$. In other words, $\tau_{2}$ is the dominant relaxation time which characterizes the critical slowing down of the staggered magnetization.
3.2. Response of the System to a Time-Varying Staggered Magnetic Field. In order to derive the kinetic equations leading to complex order parameter susceptibility, let us assume that the system is stimulated by a staggered magnetic field $H_{s}$ oscillating at an angular frequency $\omega$ while $H=H_{0}$. It is a well-known fact that all quantities will oscillate at $\omega$ at the steady state:

$$
\begin{gathered}
H_{s}-H_{s_{0}}=H_{s_{1}} e^{i \omega t}, \\
m-m_{0}=m_{1} e^{i \omega t}, \\
s-s_{0}=s_{1} e^{i \omega t} .
\end{gathered}
$$

In addition, embedding (16) into the kinetic equations (10), one obtains

$$
\begin{gathered}
\left(\gamma_{m} c+\gamma g-i \omega\right) m_{1}+\left(\gamma_{m} g+\gamma d\right) s_{1}=-\gamma k H_{s_{1}}, \\
\left(\gamma c+\gamma_{s} g\right) m_{1}+\left(\gamma g+\gamma_{s} d-i \omega\right) s_{1}=-\gamma_{s} k H_{s_{1}} .
\end{gathered}
$$

Solving (17) yields the following expression for $s_{1} / H_{s_{1}}$ :

$$
\frac{s_{1}}{H_{s_{1}}}=\frac{\left|\begin{array}{cc}
\gamma_{m} c+\gamma g-i \omega & -\gamma k \\
\gamma c+\gamma_{s} g & -\gamma_{s} k
\end{array}\right|}{\left|\begin{array}{cc}
\gamma_{m} c+\gamma g-i \omega & \gamma_{m} g+\gamma d \\
\gamma c+\gamma_{s} g & \gamma g+\gamma_{s} d-i \omega
\end{array}\right|} .
$$

The determinant in the denominator of (18) is the same as the secular determinant which is used for the calculation of the reciprocal relaxation times given by (11) except for the replacement of $\tau^{-1}$ by $i \omega$. Hence, (18) may be written as

$$
\frac{s_{1}}{H_{s_{1}}}=\frac{i \omega k \gamma_{s}-d k\left(\gamma_{m} \gamma_{s}-\gamma^{2}\right)}{\left(i \omega+\tau_{1}^{-1}\right)\left(i \omega+\tau_{2}^{-1}\right)} .
$$

Now, (19) is needed in order to calculate the complex staggered susceptibility $\chi_{s}(\omega)$. This may be seen as follows. The total induced staggered magnetic moment per unit volume of the metamagnetic Ising model is given by

$$
s-s_{\infty}=\operatorname{Re}\left\{s_{1} e^{i \omega t}\right\} .
$$

Here, $s_{\infty}$ is the staggered magnetization induced by a staggered field oscillating at $\infty$-frequency. Further, by definition, the expression for the complex staggered susceptibility reads

$$
s-s_{\infty}=\operatorname{Re}\left\{\chi_{s}(\omega) H_{s_{1}} e^{i \omega t}\right\} .
$$

Comparing (19) and (21), one may write complex staggered susceptibility as follows:

$$
\chi_{s}(\omega)=\frac{s_{1}}{H_{s_{1}}} .
$$

Finally, using the relation

$$
\chi_{s}(\omega)=\chi_{s}^{\prime}(\omega)-i \chi_{s}^{\prime \prime}(\omega),
$$


one finds

$$
\begin{aligned}
& \chi_{s}^{\prime}(\omega)=k \frac{\left(\tau_{1} \tau_{2}\right)}{\left(\tau_{2}-\tau_{1}\right)}\left(\frac{\gamma_{s}+\tau_{1} c\left(\gamma_{s} \gamma_{m}-\gamma^{2}\right)}{1+\omega^{2} \tau_{1}^{2}}\right. \\
& \left.-\frac{\gamma_{s}+\tau_{2} c\left(\gamma_{s} \gamma_{m}-\gamma^{2}\right)}{1+\omega^{2} \tau_{2}^{2}}\right), \\
& \chi_{s}^{\prime \prime}(\omega)=k \frac{\left(\tau_{1} \tau_{2}\right)}{\left(\tau_{2}-\tau_{1}\right)}\left(\frac{\omega \tau_{1}\left(\gamma_{m}+\tau_{1} c\left(\gamma_{s} \gamma_{m}-\gamma^{2}\right)\right)}{1+\omega^{2} \tau_{1}^{2}}\right. \\
& \left.-\frac{\omega \tau_{2}\left(\gamma_{m}+\tau_{2} c\left(\gamma_{s} \gamma_{m}-\gamma^{2}\right)\right)}{1+\omega^{2} \tau_{2}^{2}}\right) .
\end{aligned}
$$

$\chi_{s}^{\prime}(\omega)$ and $\chi_{s}^{\prime \prime}(\omega)$ contain a linear superposition (with a temperature dependent coefficient) of two Debye forms characterized by the two relaxation times of the metamagnetic Ising model $\left(\tau_{1}, \tau_{2}\right)$. In accordance with the fact that our analysis is based on linear response theory, the real and imaginary parts of $\chi_{s}(\omega)$ are independent of the amplitude of the timevarying staggered field $H_{s_{1}}$ but dependent on the frequency of the field $\omega$. On the other hand, physical interpretations of the real and imaginary parts of the complex susceptibility are different. The imaginary part can be expressed as $\chi_{s}^{\prime \prime}(\omega)=$ $(1 / 2)\left[\chi_{s}(\omega)-\chi_{s}^{*}(\omega)\right]$, where $\chi_{s}^{*}(\omega)$ is the complex conjugate of $\chi_{s}(\omega)$. In addition, by making use of the fact that $\chi_{s}(t)$ is the inverse Fourier transform of $\chi_{s}^{\prime \prime}(\omega)$, one obtains

$$
\begin{aligned}
\chi_{s}^{\prime \prime}(\omega) & =\frac{i}{2} \int_{-\infty}^{\infty} \chi_{s}(t)\left[e^{i \omega t}-e^{i \omega t}\right] d t \\
& =\frac{i}{2} \int_{-\infty}^{\infty} e^{i \omega t}\left[\chi_{s}(t)-\chi_{s}(-t)\right] d t
\end{aligned}
$$

Equation (25) indicates that the imaginary part of $\chi_{s}(\omega)$ is attributable to the part of dynamic response function which is not invariant under time reversal. Namely, it arises due to dissipative irreversible processes that take place in the system. Due to the fact that $\chi_{s}^{\prime \prime}(\omega)$ is the dissipative or absorptive part of the response function, it is called the absorption factor. The same analysis as above shows that the real component is given by the following equation for the real part:

$$
\chi_{s}^{\prime}(\omega)=\frac{1}{2} \int_{-\infty}^{\infty} e^{i \omega t}\left[\chi_{s}(t)-\chi_{s}(-t)\right] d t
$$

Contrary to the absorption factor, the real part is invariant under time reversal and is thus related to the reversible magnetization process and stays in phase with the oscillating staggered magnetic field. Since then, $\chi_{s}^{\prime}(\omega)$ has been called the dispersion factor or in-phase component of the AC staggered susceptibility [5].

\section{Frequency Response of the AC Order Parameter Susceptibility}

It is instructive to review the zero and $\infty$-frequency limits of the staggered dispersion and absorption factors first. The metamagnetic Ising model experiences the so-called phenomenon of critical slowing down characterized by the dominant relaxation time $\tau_{2}$ in the vacancy of the field induced Neel point [13]. Thus, $\omega \tau_{2}$ is the quantity that rules the separation of the so-called low- and high-frequency regions. In addition, since $\tau_{2}^{-1} \rightarrow 0$ as $T \rightarrow T_{N}$, one can choose to keep the frequency of the oscillating staggered field $\omega$ fixed and observe, for certain fixed frequencies, the low-frequency behaviors followed by the higher-frequency behaviors as $T \rightarrow T_{N}$. Since then, for any finite $\omega$, one can observe the high-frequency results if the temperature considered is close enough to the critical temperature $T_{N}$.

The investigation of the temperature variance of the staggered dispersion and absorption factors of the metamagnetic Ising model has shown that their critical characteristics in high- and low-frequency regions are quite different [15]. In the low-frequency regime, the AC staggered magnetization can readily follow the applied field $H_{s}$ since $\omega$ is small. In addition, due to the fact that $\tau_{2}$ varies as $\left|T-T_{N}\right|^{-1 / 2}$, if one approaches the Neel point, the low-frequency region corresponds to zero frequency limit for which $\chi_{s}(\omega)$ corresponds to the isothermal staggered susceptibility:

$$
\lim _{\omega \rightarrow 0} \chi_{s}(\omega)=\left(\frac{\partial s}{\partial H_{s}}\right)_{T}=\chi_{s_{T}} .
$$

Thus, one expects $\chi_{s}^{\prime}(\omega)$ to converge to static results and $\chi_{s}^{\prime \prime}(\omega)$ to vanish in the zero frequency limit. As the frequency grows, the staggered magnetization generated by the AC staggered field cannot change more rapidly than the spinspin relaxation time, so that the AC staggered susceptibility becomes lower and lower and approaches the adiabatic staggered susceptibility in the infinite-frequency limit $[18,19]$ :

$$
\lim _{\omega \rightarrow \infty} \chi_{s}(\omega)=\left(\frac{\partial s}{\partial H_{s}}\right)_{S}=\chi_{s_{S}} .
$$

One should note that the reduced values of the temperature and the physical and staggered magnetic fields will be utilized:

$$
\begin{gathered}
T_{r}=\frac{T}{\left|J_{1}\right|}, \\
H_{r}=\frac{H}{\left|J_{1}\right|}, \\
H_{s_{r}}=\frac{H_{s}}{\left|J_{1}\right|},
\end{gathered}
$$

from now on. Due to the fact that metamagnetic-paramagnetic phase transition takes place for zero static staggered field, $H_{s_{r}}=0$ in all figures. In light of this information, the reactive and dissipative parts of the AC staggered susceptibility spectra of the metamagnetic Ising model will be presented in the paramagnetic phase first. With this aim, $\log \chi_{s}^{\prime}(\omega)$ and $\log \chi_{s}^{\prime}(\omega)$ versus $\log \omega$ plots are given at $T_{r}=5.64$ while $r=1.0, H_{r_{N}}=1.0, T_{r_{N}}(1)=5.563$, and $\gamma_{s}=-10.0, \gamma_{m}=$ -0.010 , and $\gamma=-0.001$. We should note that the diagonal and off-diagonal coefficients of the kinetic matrix satisfy the 


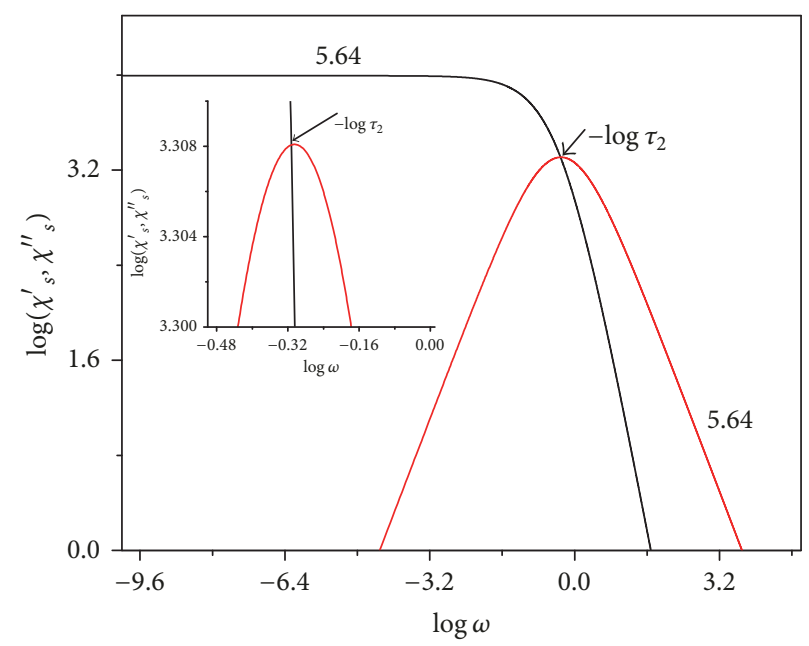

FIGURE 1: Frequency dependence of $\chi_{s}(\omega)$ in the paramagnetic phase. The number accompanying each curve is the value of the reduced temperature while $r=1.0, H_{r_{N}}=1.0, T_{r_{N}}(1)=5.563$, and $\gamma_{s}=-10.0, \gamma_{m}=-0.01$, and $\gamma=-0.001$.

condition $\gamma_{s} \gamma_{m} \gg \gamma$ which is valid through our analysis as already discussed in Section 2. Denoted by the black curve, $\chi_{s}^{\prime}(\omega)$ becomes the isothermal staggered susceptibility, whereas $\chi_{s}^{\prime \prime}(\omega)$ vanishes for $\omega \ll \tau_{2}^{-1}$. These results are in accordance with the temperature variances of $\chi_{s}^{\prime}(\omega)$ and $\chi_{s}^{\prime \prime}(\omega)$ in the low-frequency region: $\chi_{s}^{\prime}(\omega)$ follows the scaling law $\left|T_{r}-T_{r_{N}}\right|^{-1}$ for temperatures converging $T_{r_{N}}$ and $\chi_{s}^{\prime \prime}(\omega)$ varies as $\omega\left|T_{r}-T_{r_{N}}\right|^{-2}$ and vanishes in the limit $\omega \rightarrow 0$ [15]. Finally, zero frequency limit of $\chi_{s}$ is comparable with the static results of iron group dihalides in static fields where $\chi_{s_{T}}$ increases rapidly with rising temperature and diverges at the critical and multicritical points $[9,20]$.

As shown in Figure $1, \chi_{s}^{\prime \prime}(\omega)$ adopts a maximum value, $\chi_{s}^{\prime}(\omega)$ undergoes a step-like change just for $\omega=\tau_{2}^{-1}$, and $\omega \tau_{2}$ separates the so-called low- and high-frequency regions as a signature of the Debye type relaxation [18]. One can see from Figure 1 that both $\chi_{s}^{\prime}(\omega)$ and $\chi_{s}^{\prime \prime}(\omega)$ vanish as $\omega \rightarrow \infty$. This implies that $\chi_{s_{S}}=0$ for $H_{s_{r}}=0$ and is in accordance with the fact that the adiabatic susceptibility is zero for vanishing static fields [18]. Parallel with these findings, the constant frequency thermal variance of $\chi_{s}^{\prime}(\omega)$ of the metamagnetic Ising model is reported to display a local minimum equal to zero at $T_{r}=T_{r_{N}}$ and vary as $\left|T_{r}-T_{r_{N}}\right|^{+1}$ for temperatures slightly below and above $T_{r_{N}}$ for frequencies that satisfy the condition $\omega \tau_{2} \gg 1$. In addition, $\chi_{s}^{\prime}(\omega)$ has two frequency dependent local maxima in paramagnetic and antiferromagnetic phases while $\chi_{s}^{\prime \prime}(\omega)$ displays a minimum at the phase transition point whose amplitude decreases with increasing frequency [15].

Unlike the above-mentioned behavior of the AC order parameter susceptibility spectra in the paramagnetic phase, double logarithmic plots of $\chi_{s}^{\prime}(\omega)$ and $\chi_{s}^{\prime \prime}(\omega)$ versus $\omega$ exhibit two distinct characters for $\left|\gamma_{s}\right| \gg\left|\gamma_{m}\right|$ and $\gamma_{s} \approx \gamma_{m}$ in the metamagnetic phase. Figures 2(a)-2(b) display $\log \chi_{s}^{\prime}(\omega)$ and $\log \chi_{s}^{\prime \prime}(\omega)$ versus $\log \omega$ plots for these two cases while $T_{r}<$ $T_{r_{N}}$. Here, red and black curves display the logarithms of the calculated dispersion and absorption factors while $\left|\gamma_{s}\right| \gg\left|\gamma_{m}\right|$ and $\gamma_{s} \approx \gamma_{m}$, respectively. Meanwhile, the symbols $\tau_{1}, \tau_{2}$ and $\tau_{1}^{\prime}, \tau_{2}^{\prime}$ denote the two relaxation times of the system calculated for $\left|\gamma_{s}\right| \gg\left|\gamma_{m}\right|$ and $\gamma_{s} \approx \gamma_{m}$, respectively. Finally, the number accompanying each curve is the value of the reduced temperature while $r=1.0, H_{r_{N}}=1.0$, and $T_{r_{N}}(1)=5.563$.

Figure 2(a) presents the dissipative part of the order parameter susceptibility spectra in the ordered phase of the metamagnetic Ising model for both sets of the rate coefficients. The $\log \chi_{s}^{\prime \prime}(\omega)$ versus $\log \omega$ plot has one peak structure for $\gamma_{s} \approx \gamma_{m}\left(\gamma_{s}=-10.0, \gamma_{m}=-9.0\right)$ and evolves to a two-peak structure while $\left|\gamma_{s}\right| \gg\left|\gamma_{m}\right|\left(\gamma_{s}=\right.$ $\left.-10.0, \gamma_{m}=-0.01\right)$. For $\gamma_{s} \approx \gamma_{m}$, the staggered absorption factor has a single maximum at $\omega^{-1}=\tau_{2}^{\prime}$, where $\tau_{2}^{\prime}$ is the dominant relaxation time that governs the critical slowing down phenomena for $\gamma_{s} \approx \gamma_{m}$. Consequently, $\chi_{s}^{\prime \prime}$ spectrum is of Debye form and the order parameter irreversible relaxation is characterized by the single relaxation time $\tau_{2}^{\prime}$ in the ordered phase while $\gamma_{s} \approx \gamma_{m} \cdot \chi_{s}^{\prime \prime}$ spectrum has a different structure for $\left|\gamma_{s}\right| \gg\left|\gamma_{m}\right|$ : there exist two maxima in $\chi_{s}^{\prime \prime}$ for temperatures below $T_{r_{N}}$. The maximum at low frequency is located at $\omega=$ $\tau_{2}^{-1}$ while the second maximum takes place for $\omega=\tau_{1}^{-1}$. The first maximum is broader and has a greater amplitude than the second peak. Both maxima shift to lower frequencies and their heights grow with rising temperature toward $T_{r_{N}}$. Hence, the irreversible relaxation of the order parameter is a two-step process where there exist two long relaxation times characterizing the dynamics while $T_{r}<T_{r_{N}}$ and $\left|\gamma_{s}\right| \gg$ $\left|\gamma_{m}\right|$. We should note that a similar behavior is reported for various glass formers when approaching the glass transition [21]. Their relaxation is a two-step process in the supercooled liquid (ordered) phase where the correlation function first decays rather quickly to a plateau and departs from this plateau value on a much longer time scale. Parallel with this behavior of the correlation function, the absorption factor is characterized by a two-peak structure for $T<T_{g}$. This behavior is a common experimental feature and is called the stretching and shouldering of the relaxation (of, e.g., the 


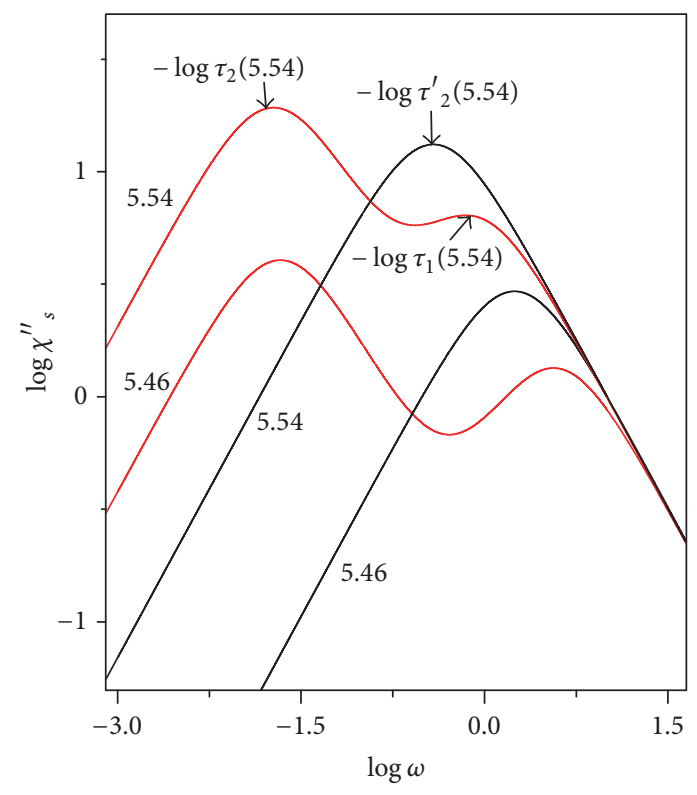

(a)
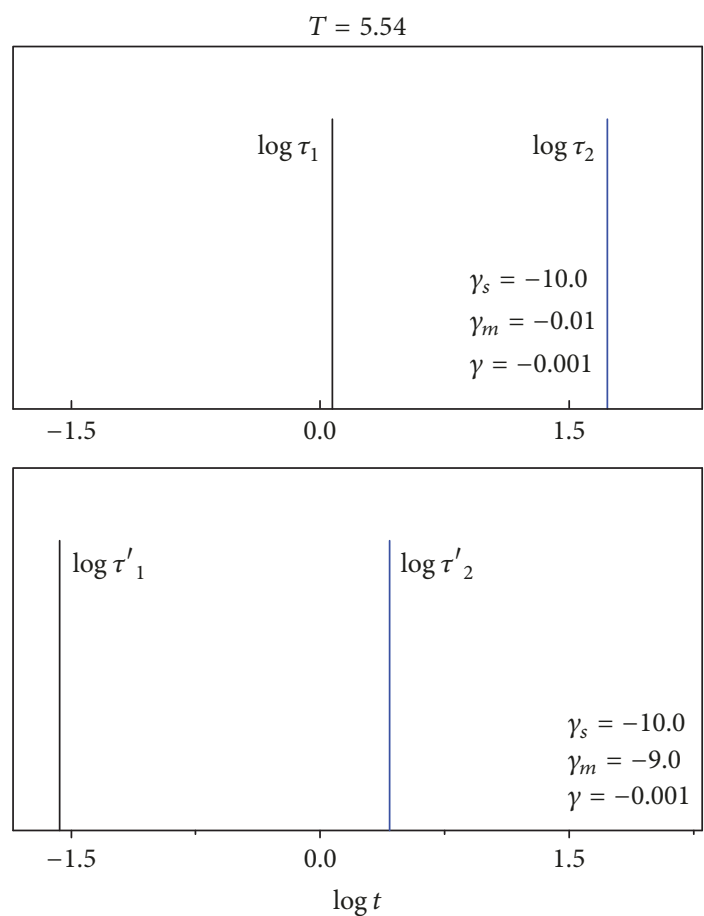

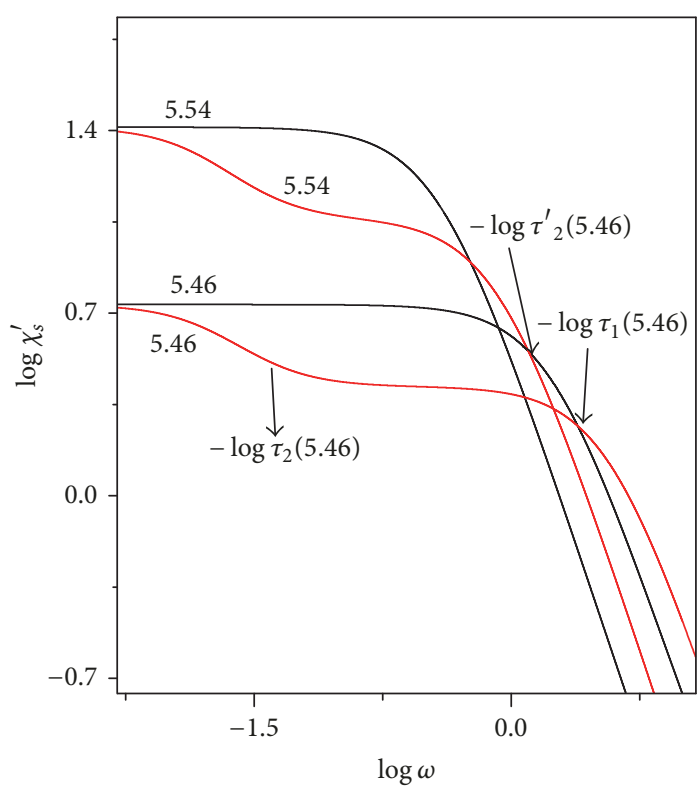

(b)
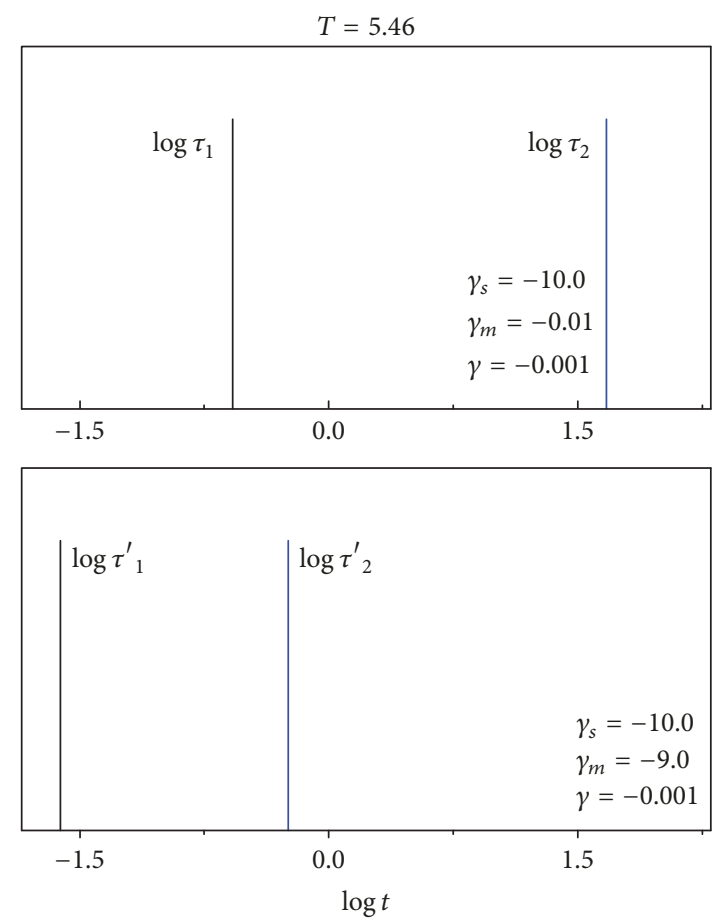

(c)

FIGURE 2: Double logarithmic plots of (a) the staggered magnetic absorption factor $\left(\chi_{s}^{\prime \prime}\right)$ and (b) the staggered dispersion factor $\left(\chi_{s}^{\prime}\right)$ versus frequency of the staggered magnetic field for two different sets of Onsager's rate coefficients (red lines for $\gamma_{s}=-10.0, \gamma_{m}=-0.01$, and $\gamma=-0.001$; black lines for $\gamma_{s}=-10.0, \gamma_{m}=-9.0$, and $\left.\gamma=-0.001\right)$ in the ferromagnetic phase. The number accompanying each curve is the value of the reduced temperature. (c) The distributions of the two relaxation times of the metamagnetic Ising model for two different sets of Onsager's rate coefficients. Here, $r=1.0$ and $H_{r_{N}}=1.0$ and $T_{r_{N}}(1)=5.563$.

density fluctuations) as the temperature is decreased toward $T_{g}$ [22]. Further, Erdem reported that the structure of the AC bipolar susceptibility spectra of the BEG model also depends on the relation between the diagonal phenomenological coefficients for temperatures below the critical temperature [16].

The reactive part of $\chi_{s}(\omega)$ spectrum in the metamagnetic phase is shown in Figure 2(b). For $\gamma_{s} \approx \gamma_{m}\left(\gamma_{s}=-10.0\right.$, 
$\left.\gamma_{m}=-9.0\right)$, the staggered dispersion factor presents a singleplateau structure which is in the same location as the corresponding maximum for the staggered absorption factor, shown in Figure 2(a). Both the plateau of $\chi_{s}^{\prime}(\omega)$ and the maximum of $\chi_{s}^{\prime \prime}(\omega)$ stay localized in the vicinity of $\omega \simeq$ $\tau_{2}^{\prime-1}$. In addition, the staggered dispersion factor becomes the isothermal staggered susceptibility for $\omega \gg \tau_{2}^{\prime-1}$. These behaviors imply the existence of Debye type reversible magnetization relaxation of the metamagnetic Ising model for $\gamma_{s} \approx \gamma_{m}$. On the other hand, two plateau regions are obtained in $\log \chi_{s}^{\prime}$ versus $\log \omega$ plots in which the higher plateau satisfies the condition $\omega \ll \tau_{2}^{-1}$ for all frequency values for $\left|\gamma_{s}\right| \gg\left|\gamma_{m}\right|$. The single plateau of the dispersion factor calculated for $\left|\gamma_{s}\right| \simeq\left|\gamma_{m}\right|$ and the higher plateau of the dispersion factor calculated for $\left|\gamma_{s}\right| \gg\left|\gamma_{m}\right|$ overlap in the lowfrequency region. In other words, the low-frequency limit of staggered dispersion factor converges to the isothermal susceptibility for both sets of Onsager rate coefficients as expected. The height of the higher plateau grows rapidly as one approaches the critical point in accordance with the fact that isothermal ordering susceptibility diverges as $\mid T_{r}-$ $T_{r_{N}} \mid \rightarrow 0$. The second plateau starts at $\omega \simeq \tau_{2}$ and demonstrates the reversible processes that are shorter than $\tau_{2}$. Further, the lower plateau terminates as $\omega \simeq \tau_{1}$ and the staggered dispersion factor decreases with rising frequency. In conclusion, the two maxima in $\chi_{s}^{\prime \prime}(\omega)$ and two plateaus in $\chi_{s}^{\prime}(\omega)$ indicate that both irreversible and reversible relaxation processes of the order parameter are two-step processes which are characterized by two distinct time scales at the ordered phase while $\left|\gamma_{s}\right| \gg\left|\gamma_{m}\right|$.

The spectrum of the relaxation times that is obtained by utilizing (13) is given in Figure 2(c) for two different temperatures that are below $T_{r_{N}}$. The spectra calculated for $\gamma_{s}=-10.0$ and $\gamma_{m}=-9.0\left(\left|\gamma_{s}\right| \approx\left|\gamma_{m}\right|\right.$ case $)$ which is given at the bottom for both temperatures indicate that $\tau_{1}^{\prime}$ is much lower than $\tau_{2}^{\prime}$. This is in accordance with the single-peak and single-plateau structure of $\chi_{s}^{\prime \prime}(\omega)$ and $\chi_{s}^{\prime}(\omega)$ spectra discussed above, which implies that the Debye type relaxation characterized a single long relaxation time $\tau_{2}^{\prime}$. On the other hand, a comparison between the above and bottom panels of Figure 2(c) reveals the fact that $\tau_{1}$ which is calculated for $\gamma_{s}=-10.0$ and $\gamma_{m}=-0.010$ is quite larger than $\tau_{1}^{\prime}$ and nearly of the same order of magnitude as $\tau_{2}^{\prime}$ for both temperatures below $T_{r_{N}}$. Thus, in the metamagnetic phase, there exist two long and noticeably separated relaxation times for only $\left|\gamma_{s}\right| \gg\left|\gamma_{m}\right|$. The gaps in the spectrum of the relaxation times are shown to affect both the reactive and the dissipative parts of the AC susceptibility spectra by making use of Glauber dynamics for various systems: spin-glass systems [23], frustrated semimagnetic semiconductors [24], Isingspin clusters in random fields [25], and Ising spins with AF bonds [26]. In these studies, the existence of the gaps in the spectrum of the relaxation times obtained within the Glauber dynamics which caused the real part of the frequency dependent susceptibility reveals a sequence of plateaus as a function of $\log \omega$, whereas the imaginary part displays a sequence of maxima. The number of long relaxation times is the only difference relative to these theoretical systems which is observed in the frequency dependent $\mathrm{AC}$ susceptibility spectra. For instance, the magnetic relaxation of the AF-F and F-AF spin clusters given in [26] is characterized by six long relaxation times. The largest diverging relaxation time is separated from the other five relaxation times that take very close values and act like a single relaxation time in logarithmic scale. It is reported that the diverging relaxation times can either be close to each other on a logarithmic scale or be noticeably separated depending on both the exchange couplings and the temperature [23-26]. In conclusion, the existence of two long and noticeably separated relaxation times is the underlying reason of the two-peak structure in $\chi_{s}^{\prime \prime}$ and two plateaus of $\chi_{s}^{\prime}$ metamagnetic Ising model while $\left|\gamma_{s}\right| \gg\left|\gamma_{m}\right|$ and $T_{r}<T_{r_{N}}$.

Figures 3(a)-3(c) present the behavior of the order parameter relaxation near the field induced Neel point for $r=$ $1.0, H_{r_{N}}=1.0$, and $T_{r_{N}}=5.563$. The frequency dependencies of $\chi_{s}^{\prime \prime}(\omega)$ and $\chi_{s}^{\prime}(\omega)$ as well as the spectrum of the relaxation times for temperatures above and below the second-order phase transition point are shown in Figures 3(a)-3(c). As the temperature converges to critical temperature from both sides, $\chi_{s}^{\prime \prime}$ has a single peak and $\chi_{s}^{\prime}$ undergoes one step-like change which occurs when the frequency of the perturbation field is equal to the reciprocal diverging relaxation time both for $\left|\gamma_{s}\right| \gg\left|\gamma_{m}\right|$ and for $\left|\gamma_{s}\right| \simeq\left|\gamma_{m}\right|$. The staggered absorption factor approaches zero with increasing frequency in the highfrequency regime and its zero frequency limit converges to $\chi_{s_{T}}$ which diverges as $\left|T_{r} \rightarrow T_{r_{N}}\right|$. The plots for $T_{r}=5.5640>$ $T_{r_{N}}$ show that a selection of the Onsager rate coefficients lose their impact on the relaxation dynamics of the staggered magnetization as one enters the disordered phase. Finally, Figure 3(c) which is obtained by making use of (13) denotes that $\tau_{1}$, the first relaxation time calculated for $\left|\gamma_{s}\right| \gg\left|\gamma_{m}\right|$, takes much higher values than its correspondence $\tau_{1}^{\prime}$ which is calculated for $\left|\gamma_{s}\right| \simeq\left|\gamma_{m}\right|$. Hence, the metamagnetic Ising model has still two long relaxation times for $\gamma_{s}=-10.0, \gamma_{m}=$ -0.01 , and $\gamma=-0.001$, but they are no longer noticeably separated in logarithmic scale and behave like a single relaxation time in the vicinity of the critical point. Both reversible and irreversible relaxation dynamics of the order parameter are governed by the single diverging relaxation time of the system as $\left|T_{r}-T_{r_{N}}\right| \rightarrow 0$. Thus, AC order parameter susceptibility spectra of the metamagnetic Ising model are of Debye form and the relaxation of the staggered magnetization is a simple Debye exponential as one approaches the critical point from both sides. According to the theory of irreversible thermodynamics, the magnetic relaxation of the BEG model behaves in a similar manner in the vicinity of its critical point [16]. Further, the loss component of the frequency dependent magnetic response function of spin glasses and other glassy systems such as polymer glasses [27] and supercooled liquids evolves from a two-peak structure to a single-peak, highfrequency structure as the temperature rises toward critical temperature $T_{g}$ [22]. More precisely, the order parameter relaxation evolves from a two-step process at a lower temperature (supercooled liquid), to a simple Debye exponential at high temperatures (liquid) [21].

In order to complete the investigation of the frequency variance of the AC staggered susceptibility of a metamagnetic 

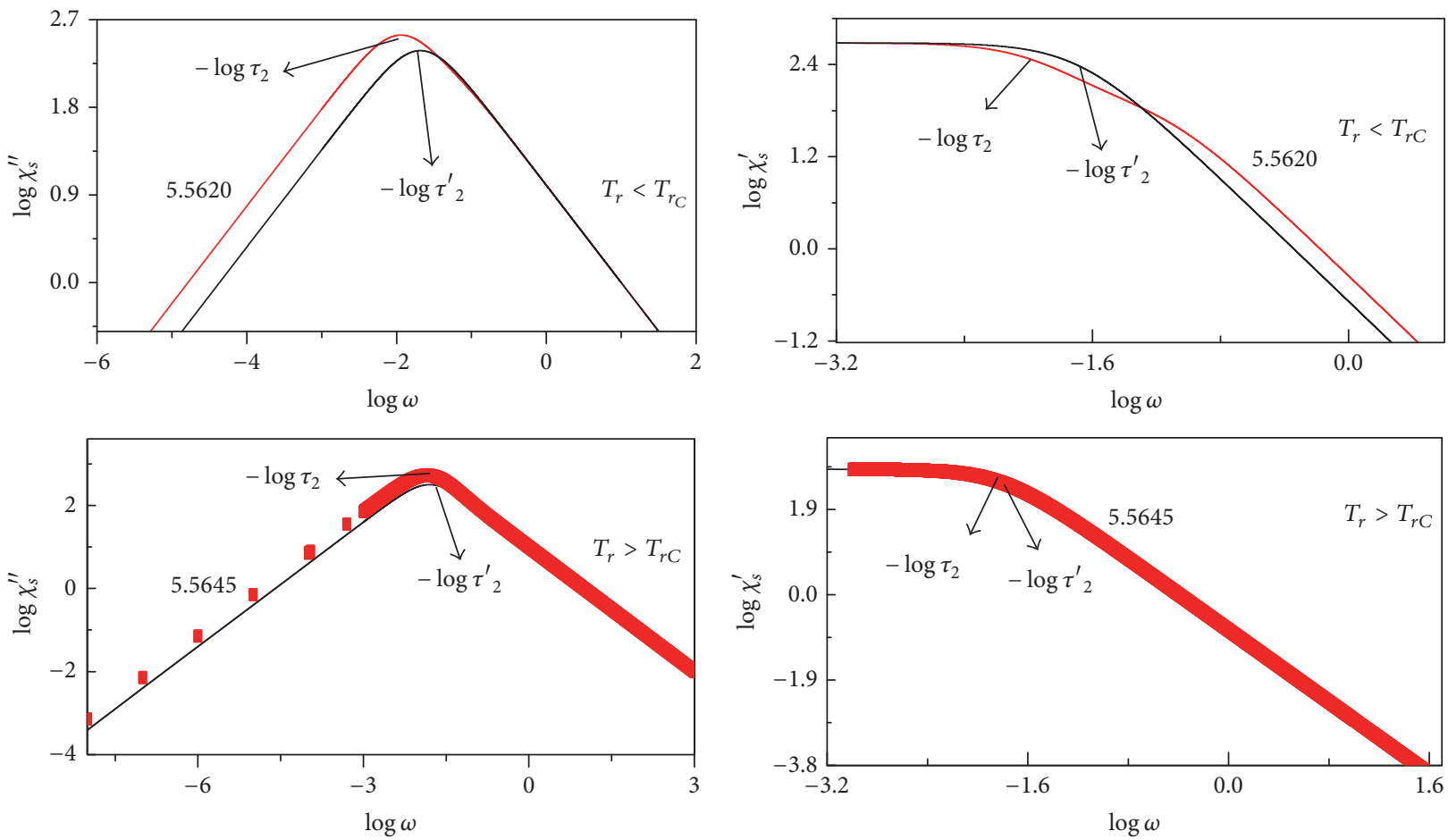

(a)

(b)
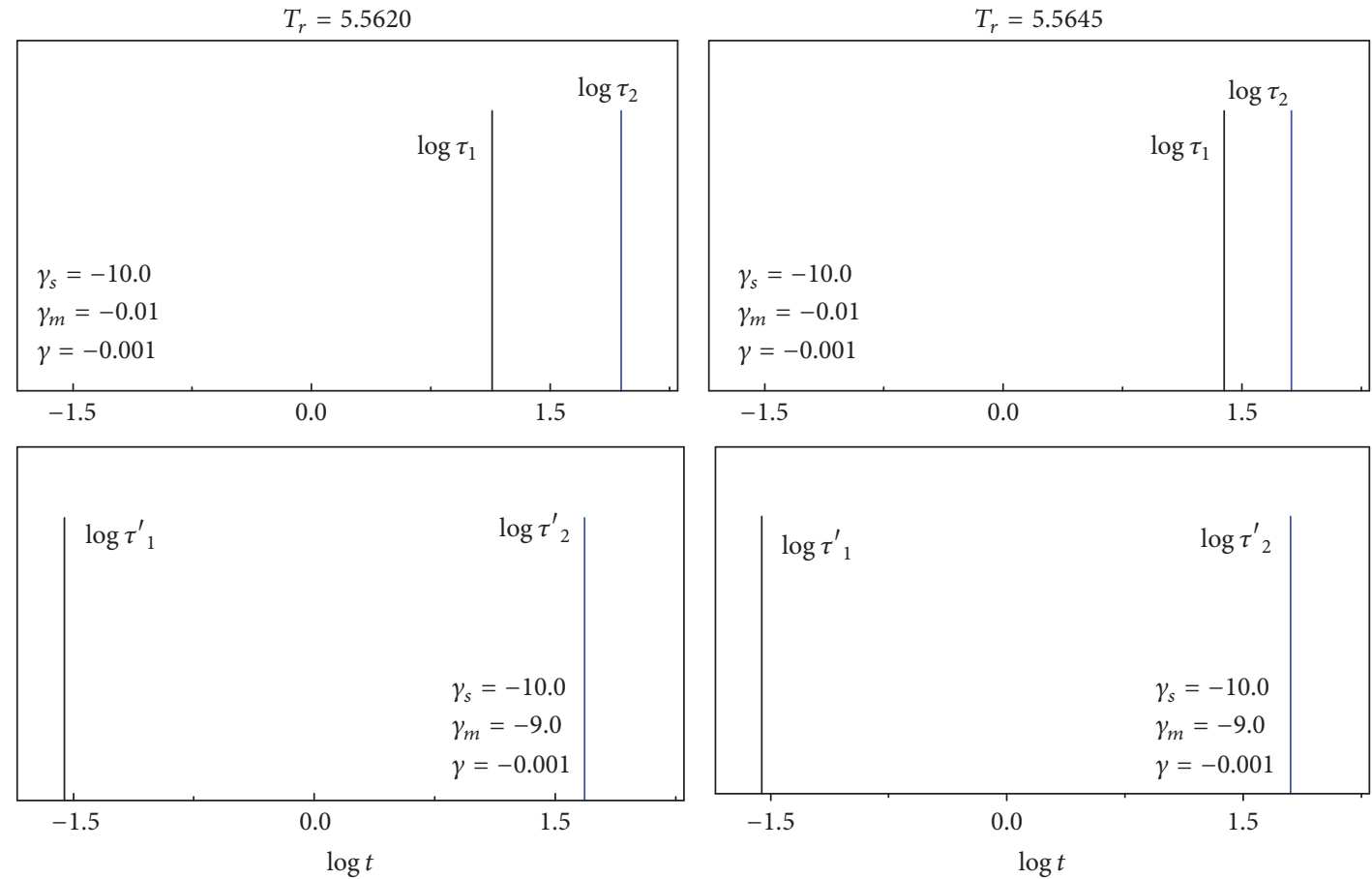

(c)

FIgURE 3: Same as Figures 2(a)-2(c) but for two distinct temperatures in the neighborhood of the critical temperature.

Ising model, an analysis of the real and imaginary parts of $\chi_{s}(\omega)$ has been elucidated using Cole-Cole plot of $\chi_{s}(\omega)$. Figures 4(a)-4(b) present $\chi_{s}^{\prime \prime}-\chi_{s}^{\prime}$ plots while $\left|\gamma_{s}\right| \gg\left|\gamma_{m}\right|$ for temperatures that correspond to ordered and disordered phases, respectively. In the ordered phase, Cole-Cole plots for the metamagnetic Ising model consist of two arcs where the larger arc corresponds to the longest relaxation process. In addition, since a sequence of the arcs in $\chi_{s}^{\prime \prime}-\chi_{s}^{\prime}$ plots tell that the relaxation process is governed by a distribution of relaxation times [5], it is clear that magnetic relaxation 


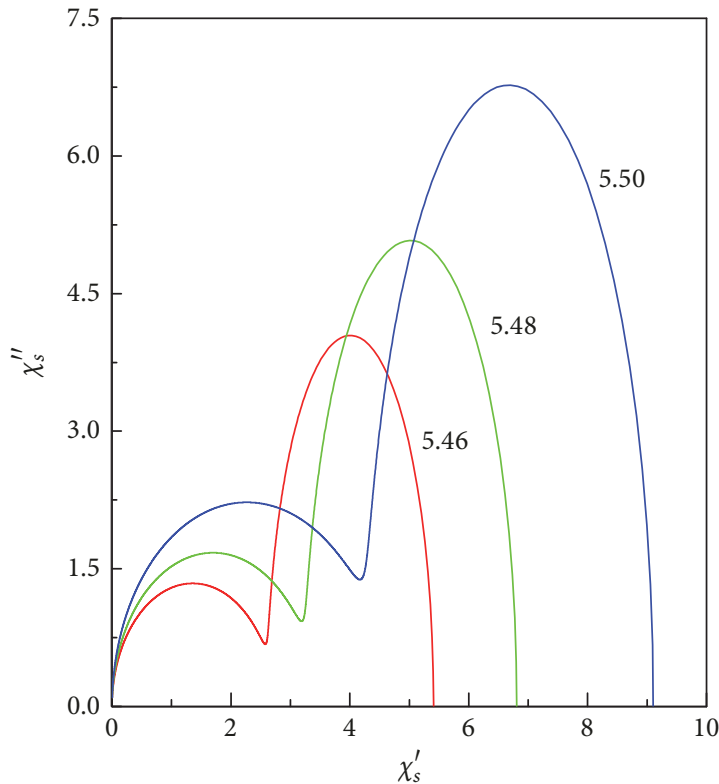

(a)

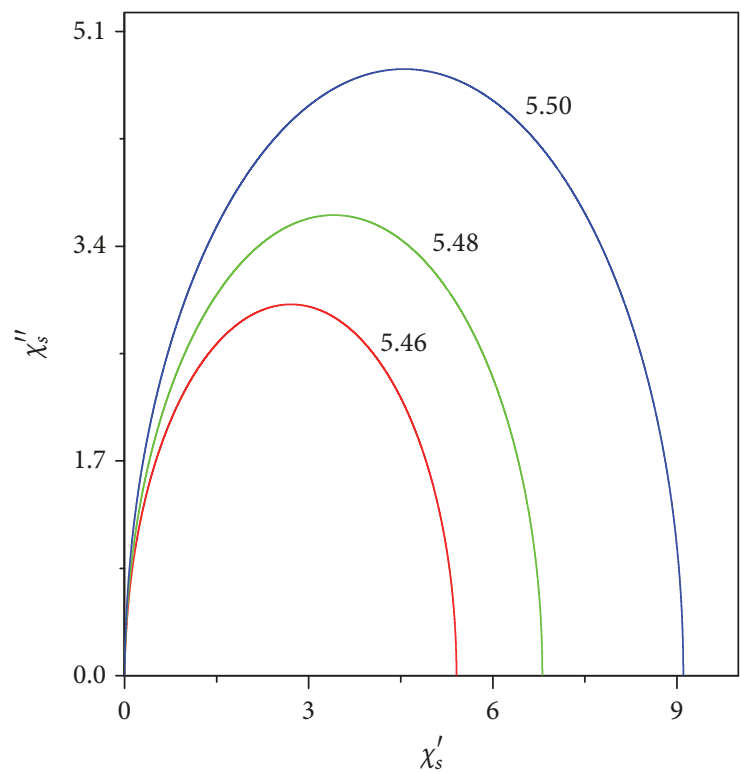

(c)

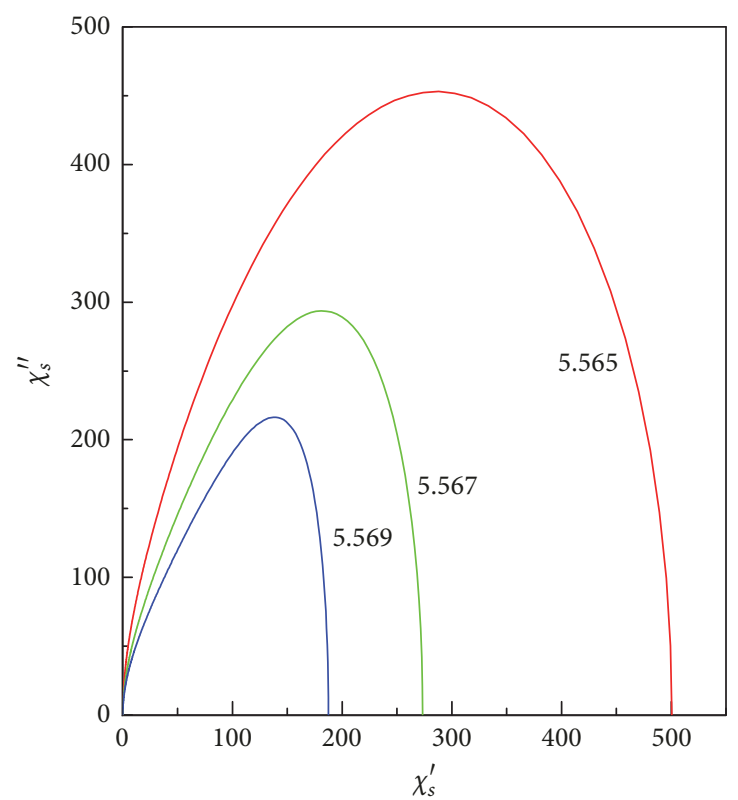

(b)

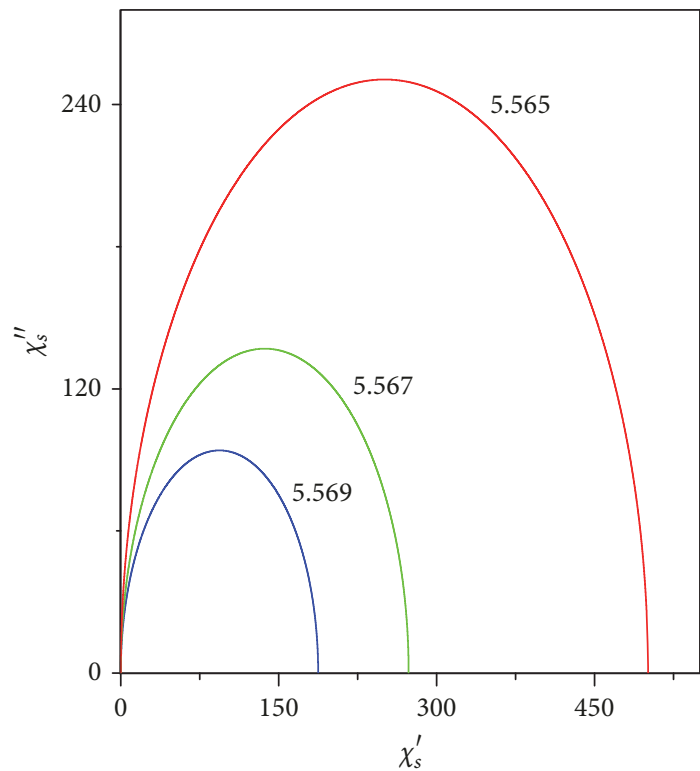

(d)

FIGURE 4: Cole-Cole plots of $\chi_{s}(\omega)$ in the (a) metamagnetic phase and (b) paramagnetic phase while $\gamma_{s}=-10.0, \gamma_{m}=-0.01$, and $\gamma=-0.001$. Cole-Cole plots of $\chi_{s}(\omega)$ in the (c) metamagnetic phase and (b) paramagnetic phase for $\gamma_{s}=-10.0, \gamma_{m}=-9.0$, and $\gamma=-0.001$. The number accompanying each curve is the value of the reduced temperature and $r=1.0, H_{r_{N}}=1.0$, and $T_{r_{N}}(1)=5.563$.

is governed by two long relaxation times for $\left|\gamma_{s}\right| \gg\left|\gamma_{m}\right|$ and $T_{r}<T_{r_{N}}$. A semicircle is obtained for temperatures above $T_{r_{N}}$ implying that paramagnetic magnetic relaxation is governed by a single relaxation time (see Figure $4(\mathrm{~b})$ ). In accordance with the fact that real and imaginary parts of the AC staggered susceptibility grow rapidly as $\left|T_{r} \rightarrow T_{r_{N}}\right|$ in lowfrequency region, all semicircles enlarge as one approaches the critical temperature from both sides. Finally, Figures 4(c)4(d) demonstrate the Cole-Cole plots of $\chi_{s}(\omega)$ in the ordered and disordered phases, respectively, while $\gamma_{s}=-10.0, \gamma_{m}=$ -9.0 , and $\gamma=-0.001$. It is clear from these figures which consist of a single semicircle in both metamagnetic and paramagnetic phases that the relaxation dynamics of the staggered magnetization are a simple Debye process for $\left|\gamma_{s}\right| \simeq\left|\gamma_{m}\right|$.

\section{Concluding Remarks}

The relaxation analysis based on the frequency dependent order parameter susceptibility of the metamagnetic Ising model is performed by means of a method where the equilibrium statistical theory and thermodynamics of irreversible processes are combined. The kinetic equations governing 
the total and staggered magnetization relaxation are derived by introducing the phenomenological rate coefficients that satisfy Onsager's reciprocal relation. After obtaining the two relaxation times that characterize the order parameter relaxation, the steady-state behavior of the order parameter is studied assuming that the system is under the effect of an oscillating staggered magnetic field and an expression is obtained for the order parameter susceptibility $\chi_{s}(\omega)$ which only depends on the frequency of the perturbation field.

The detailed investigation of $\chi_{s}(\omega)$ spectra and Cole-Cole analysis has shown that the nature of the order parameter relaxation of the metamagnetic Ising model depends on both the temperature and the relation between diagonal rate coefficients. In the paramagnetic phase, $\chi_{s}^{\prime \prime}(\omega)$ which is related to the density of relaxation times displays single maxima for all values of the diagonal elements of the matrix of kinetic coefficients $\left(\gamma_{s}, \gamma_{m}\right)$. This implies that the order parameter relaxation is always of Debye exponential for $T_{r}>T_{r_{N}}$. On the contrary, the order parameter relaxation of the metamagnetic Ising model is a two-step process for temperatures below the Neel point only for $\left|\gamma_{s}\right| \gg\left|\gamma_{m}\right|$. In short, the relaxation evolves from a simple Debye exponential at high temperatures to a two-step process at lower temperatures in which there exist two long relaxation times characterizing the relaxation of staggered magnetization while $\left|\gamma_{s}\right| \gg\left|\gamma_{m}\right|$. Similar findings are reported for model systems such as two-state random Potts spin model where the spin exchange is assumed to be random with frustration between $\mathrm{F}$ and $\mathrm{AF}$ values [28], Isingspin clusters systems on a triangular lattice with AF nearest neighbor and F next-nearest neighbor interactions [26], and experimental systems for temperatures near the critical point [29-31]. Finally, it should be emphasized that it is assumed in this study that the rate coefficients have negligible temperature dependence. The validity of this assumption should be testified by experiments.

\section{Data Availability}

The data used to support the findings of this study are available from the corresponding author upon request.

\section{Conflicts of Interest}

The author declares that there are no conflicts of interest regarding the publication of this paper.

\section{References}

[1] C. Balz, B. Lake, J. Reuther et al., "Physical realization of a quantum spin liquid based on a complex frustration mechanism," Nature Physics, vol. 12, no. 10, pp. 942-949, 2016.

[2] S. A. Grigera, R. A. Borzi, A. P. Mackenzie, S. R. Julian, R. S. Perry, and Y. Maeno, "Angular dependence of the magnetic susceptibility in the itinerant metamagnet $\mathrm{Sr}_{3} \mathrm{Ru}_{2} \mathrm{O}_{7}$," Physical Review B: Condensed Matter and Materials Physics, vol. 67, no. 21, 2003.

[3] S. Dhar, O. Brandt, A. Trampert, K. J. Friedland, Y. J. Sun, and K. H. Ploog, "Observation of spin-glass behavior in homogeneous (Ga,Mn)N layers grown by reactive molecular-beam epitaxy,"
Physical Review B: Condensed Matter and Materials Physics, vol. 67, no. 16, 2003.

[4] A. Mauger, J. Ferré, M. Ayadi, and P. Nordblad, "Dynamics of the spin-glass freezing in $\mathrm{Cd}_{0.6} \mathrm{Mn}_{0.4}$ Te," Physical Review B: Condensed Matter and Materials Physics, vol. 37, no. 15, pp. 9022-9028, 1988.

[5] M. Bałanda, "AC susceptibility studies of phase transitions and magnetic relaxation: Conventional, molecular and low-dimensional magnets," Acta Physica Polonica A, vol. 124, no. 6, pp. 964-976, 2013.

[6] L. Lundgren, P. Svedlindh, and O. Beckman, "Measurement of complex susceptibility on a metallic spin glass with broad relaxation spectrum," Journal of Magnetism and Magnetic Materials, vol. 25, no. 1, pp. 33-38, 1981.

[7] L. Lundgren, P. Svedlindh, P. Nordblad, and O. Beckman, "Dynamics of the relaxation-time spectrum in a CuMn spin-glass," Physical Review Letters, vol. 51, no. 10, pp. 911-914, 1983.

[8] A. P. Murani, "Spectral distribution of relaxation times in spin glasses," Journal of Magnetism and Magnetic Materials, vol. 22, no. 3, pp. 271-281, 1981.

[9] J. M. Kincaid and E. G. D. Cohen, "Phase diagrams of liquid helium mixtures and metamagnets: Experiment and mean field theory," Physics Reports, vol. 22, no. 2, pp. 57-143, 1975.

[10] A. J. Millis, A. J. Schofield, G. G. Lonzarich, and S. A. Grigera, "Metamagnetic quantum criticality in metals," Physical Review Letters, vol. 88, no. 21, Article ID 217204, pp. 2172041-2172044, 2002.

[11] V. M. Kalita, A. F. Lozenko, S. M. Ryabchenko, and P. A. Trotsenko, "Magnetoelasticity and domain structure in antiferromagnetic crystals of the iron-group dihalides," Low Temperature Physics, vol. 31, no. 8-9, pp. 794-806, 2005.

[12] W. Selke, "Anomalies in Ising metamagnets," Zeitschrift für Physik B Condensed Matter, vol. 101, no. 1, pp. 145-150, 1996.

[13] G. Gulpinar, D. Demirhan, and F. Buyukkilic, "Relaxation dynamics of iron-group dihalides," Physical Review E: Statistical, Nonlinear, and Soft Matter Physics, vol. 75, no. 2, Article ID 021104, 2007.

[14] G. Gulpinar and Y. Karaaslan, "Investigation of the effect of the off-diagonal Onsager rate coefficient on the relaxation dynamics of anhydrous dihalides of iron-group elements," Physics Letters A, vol. 375, no. 6, pp. 978-983, 2011.

[15] G. Gulpinar and E. Vatansever, "Critical behavior of AC antiferromagnetic and ferromagnetic susceptibilities of a spin- 1/2 metamagnetic Ising system," Journal of Magnetism and Magnetic Materials, vol. 324, no. 6, pp. 983-990, 2012.

[16] R. Erdem, "Frequency dependence of the complex susceptibility for a spin-1 Ising model," Journal of Magnetism and Magnetic Materials, vol. 321, no. 17, pp. 2592-2595, 2009.

[17] L. Onsager, "Reciprocal relations in irreversible processes. I," Physical Review A: Atomic, Molecular and Optical Physics, vol. 37, no. 4, pp. 405-426, 1931.

[18] R. Boča, "Mean and differential magnetic susceptibilities in metal complexes," Coordination Chemistry Reviews, vol. 173, no. 1, pp. 167-283, 1998.

[19] H. Yano, H. Kondo, T. Suzuki et al., "Magnetization and ac susceptibility of hcp solid ${ }^{3} \mathrm{He}$ near the nuclear ordering temperature," Physical Review Letters, vol. 65, no. 27, pp. 3401-3404, 1990.

[20] G. Gulpinar and E. Vatansever, "Anomalies in the multicritical behavior of staggered magnetic and direct magnetic susceptibilities of iron group dihalides," Journal of Magnetism and Magnetic Materials, vol. 324, no. 22, pp. 3784-3790, 2012. 
[21] J. P. Bouchaud, L. F. Cugliandolo, J. Kurchan, and M. Mezard, "Out of Equilibrium Dynamics in spin-glasses and other glassy systems, Spin-glasses and random fields," in World Scientific, P. Young, Ed., Spin-glasses and random, 1997.

[22] W. Götze and L. Sjögren, "Relaxation processes in supercooled liquids," Reports on Progress in Physics, vol. 55, no. 3, article no. 001, pp. 241-376, 1992.

[23] M. Cieplak, "Dynamical susceptibility of frustrated spin clusters," Journal of Physics C: Solid State Physics, vol. 19, no. 26, pp. 5253-5275, 1986.

[24] M. Cieplak, M. Z. Cieplak, and J. Lusakowski, "Dynamic spin susceptibility of semimagnetic semiconductors," Physical Review B: Condensed Matter and Materials Physics, vol. 36, no. 1, pp. 620-629, 1987.

[25] G. Ismail, "Dynamic Susceptibility for Ising-Spin Clusters in Random Fields," Physica Status Solidi (b) - Basic Solid State Physics, vol. 201, no. 1, pp. 277-284, 1997.

[26] G. Ismail and A. Salem, "Dynamics of ising spins with antiferromagnetic bonds on a triangular lattice," Physica Status Solidi (b) - Basic Solid State Physics, vol. 237, no. 2, pp. 530-539, 2003.

[27] K. Fukao and D. Tahara, "Aging dynamics in the polymer glass of poly(2-chlorostyrene): Dielectric susceptibility and volume," Physical Review E: Statistical, Nonlinear, and Soft Matter Physics, vol. 80, no. 5, Article ID 051802, 2009.

[28] G. Ismail and K. Bannora, "Dynamics of random one-dimensional $\pm \mathrm{J}$ systems," Czechoslovak Journal of Physics, vol. 53, no. 12, pp. 1229-1243, 2003.

[29] N. Petzold et al., "Evolution of the dynamic susceptibility in molecular glass formers: results from light scattering, dielectric spectroscopy, and NMR," The Journal of Chemical Physics, vol. 138, Article ID 12A510, pp. 1-15, 2013.

[30] A. Brodin and E. A. Rössler, "Universal and non-universal features of the dynamic susceptibility of supercooled liquids," Journal of Physics: Condensed Matter, vol. 18, no. 37, article no. 007, pp. 8481-8492, 2006.

[31] J. Gabriel, F. Pabst, and T. Blochowicz, "Debye Process and $\beta$ Relaxation in 1-Propanol Probed by Dielectric Spectroscopy and Depolarized Dynamic Light Scattering," The Journal of Physical Chemistry B, vol. 121, no. 37, pp. 8847-8853, 2017. 

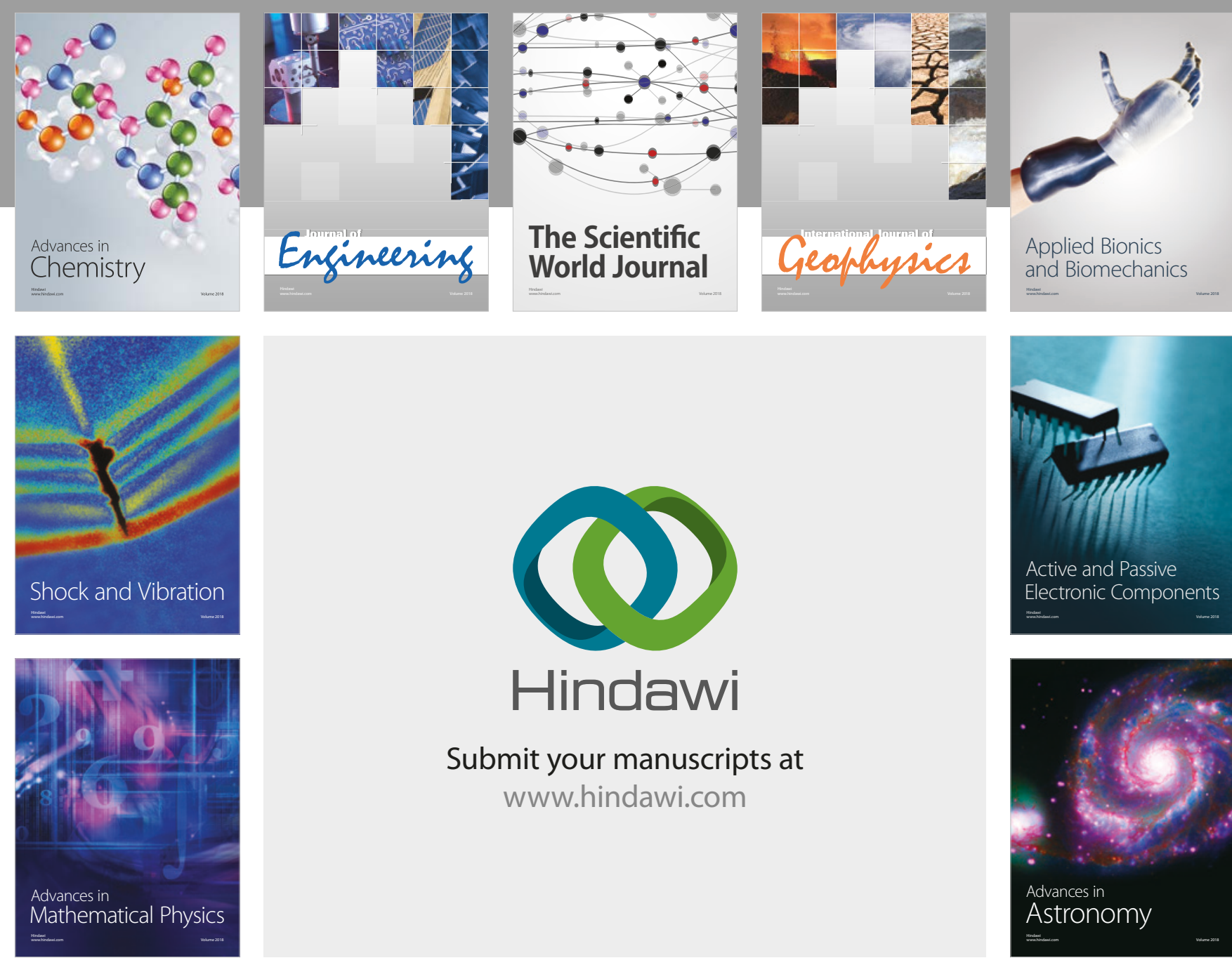

Submit your manuscripts at

www.hindawi.com

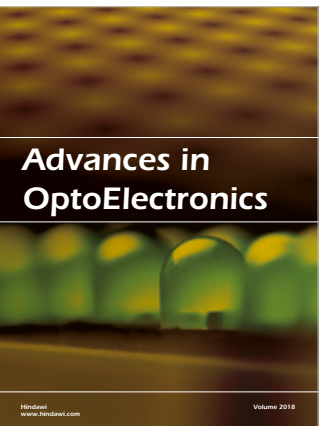

\section{Rotcting Machinery}
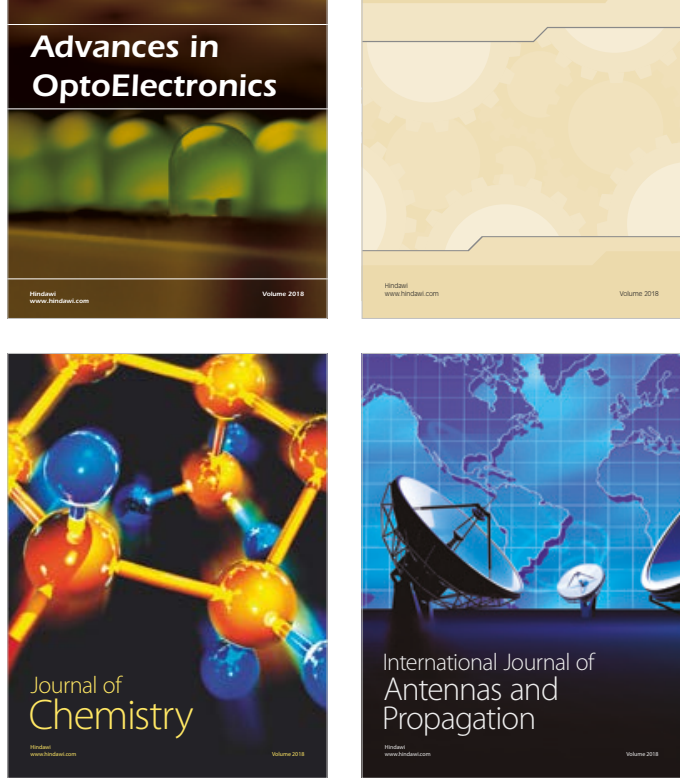

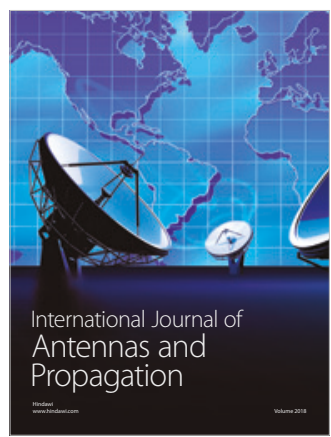

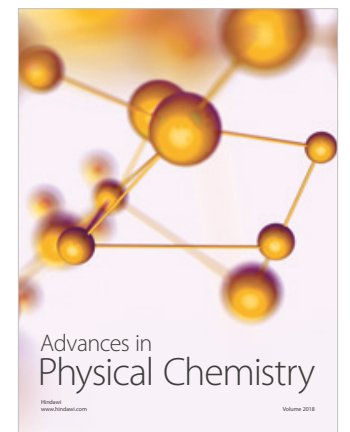

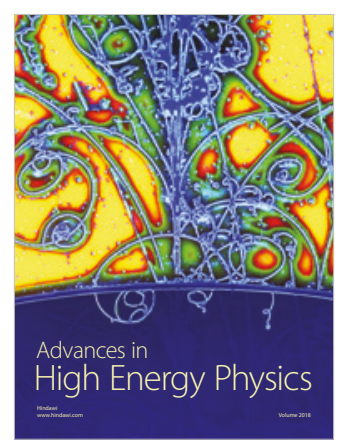

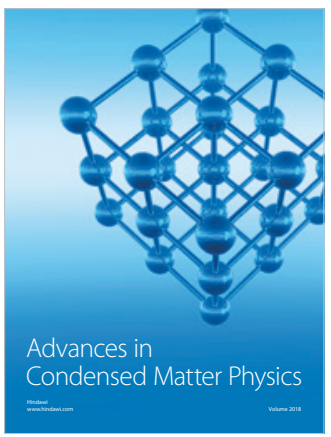

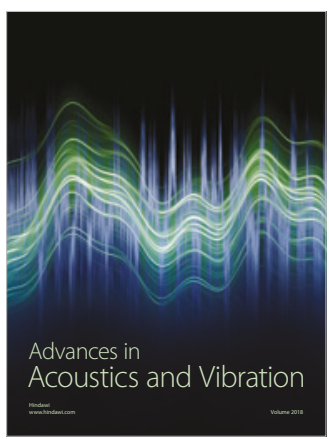

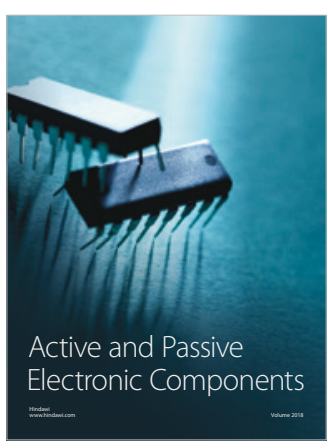
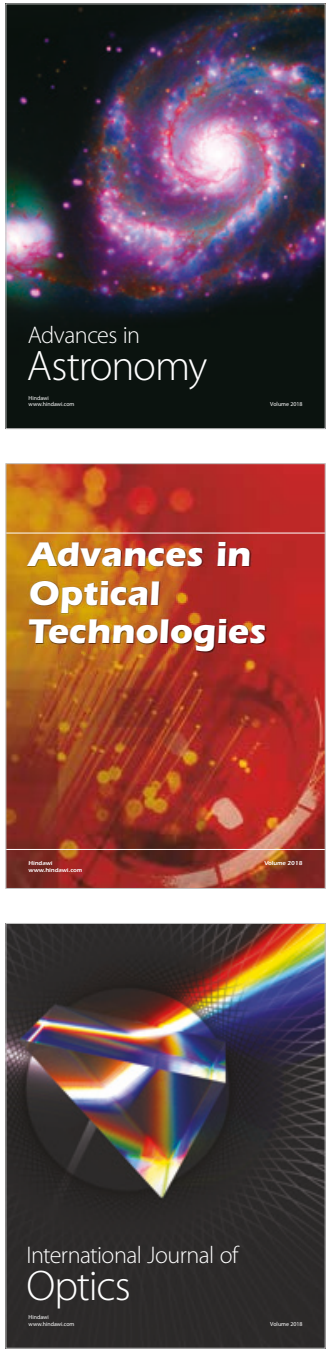